\title{
Surface fatigue crack growth suppression in cylindrical rods by artificial infiltration
}

\author{
C.S. Shin *, C.Q. Cai \\ Department of Mechanical Engineering, National Taiwan University, No. 1, Sec. 4, Roosevelt Road, Taipei 10617, Taiwan, ROC
}

Received 15 January 2007; received in revised form 2 March 2007; accepted 4 March 2007

Available online 20 March 2007

\begin{abstract}
Infiltrating foreign materials into a fatigue crack has been attempted in a number of works to suppress the progress of the crack. Previous works have been limited to through-the-thickness cracks in standard laboratory specimens. By forcing in from one surface and letting out from the other, it is relatively easier to transport foreign materials into a through-thickness crack. Part-through surface cracks are more common in practice. It will be more difficult to bring about a good infiltrant penetration to ensure effective crack growth suppression. In the current work, a special fixture has been developed to induce polymeric resin into a surface fatigue crack in a cylindrical rod. A good infiltrant penetration and crack growth retardation of more than one order of magnitude have been achieved. The relative merits of using the conventional crack closure concept and the one based on Paris et al.'s partial crack closure model to predict the resulting retardation have been investigated.
\end{abstract}

(C) 2007 Elsevier Ltd. All rights reserved.

Keywords: Fatigue crack growth retardation; Infiltration; Surface crack; Fatigue damage repair; Partial crack closure

\section{Introduction}

Fatigue fracture accounts for a great number of mechanical failures. When a fatigue crack is discovered in a critical component during routine maintenance, replacement of the component may not be feasible as there may not be stocks or the available downtime is too limited. Some expeditious provisory fixing may then be needed to allow the component to operate safely until more elaborate repair or replacement can be arranged. It has been proposed that fatigue crack growth can be slowed down or even halted by deliberately infiltrating foreign materials into a crack $[1,2]$. A number of works has been carried out to investigate the influence of materials and infiltration techniques on the efficiency of fatigue life extension [3-10]. Besides these empirical and phenomenological studies, there were also some attempts to model

\footnotetext{
* Corresponding author. Tel./fax: +88622362 2160.

E-mail address: csshin@ntu.edu.tw (C.S. Shin).
}

the process and predict the outcome [11-14]. However, all the works involved so far have been limited to through-the-thickness cracks in standard laboratory specimens. By forcing in from one surface and letting out from the other, it is relatively easier to transport foreign materials into a through-thickness crack. In practice, service fatigue cracks are often part-through and three-dimensional in nature. It will be more difficult to bring about a good infiltrant penetration to ensure effective crack growth suppression. The current work attempted to suppress the growth of a surface fatigue crack in a cylindrical rod. Such cracks are common in shafts, pins, bolts and reinforcement wires. A good infiltrant penetration and crack growth retardation of more than one order of magnitude has been achieved. The possibility of using the crack closure concept to predict the resulting retardation has also been investigated. Both the conventional crack closure model based on crack opening measurement and Paris et al.'s partial crack closure model have been compared. 


\section{Experimental procedures}

Cylindrical specimens $(15 \mathrm{~mm}$ in diameter and $200 \mathrm{~mm}$ long) were machined from a AISI 304 stainless steel rod stock. At the centre of each rod specimen, a roughly semi-elliptical surface starter notch was created by electric-discharge machining using a $0.01 \mathrm{~mm}$ thick circular copper foil electrode. The diameters of the electrode were chosen to be equal to the rod diameter $(D)$. Before testing, all specimens were stress relieved by a solution annealing treatment in vacuum at $1050{ }^{\circ} \mathrm{C}$ for one hour followed by quick cooling in a continuous supply of argon stream.

Cyclic testing was carried out on a servo-hydraulic machine with constant loading sinusoidal waveform at a frequency of $15 \mathrm{~Hz}$. The load amplitude was $20 \mathrm{kN}$ and the load ratio (min. load/max. load) was 0 . Surface crack length was monitored with a rotary traveling microscope to a resolution of $0.026 \mathrm{~mm}$. A surface crack length-compliance method [15] has been employed to monitor the crack shape development. Essentially it involved deducing the crack shape from a combination of the crack arc-length on the rod surface and the crack area. The crack area was in turn deduced from the back-face compliance. Crack growth rates were obtained from the resulting crack length-number of cycle data via a 7-point quadratic fit method. The stress intensity factor for any crack configuration was calculated by the equations reported in Ref. [16].

The compliance method aided with an offset procedure was employed to measure crack closure. A strain gage straddling the crack at the tip region was used to reflect the crack closure on the surface. A back face gage, sticking in the middle of the uncracked side, was used to reflect the bulk closure. Relative positions of these strain gages as well as nomenclature relating to the crack geometry are shown in Fig. 1. This bulk closure is more representative of the closure in the interior. Specimen compliance is measured

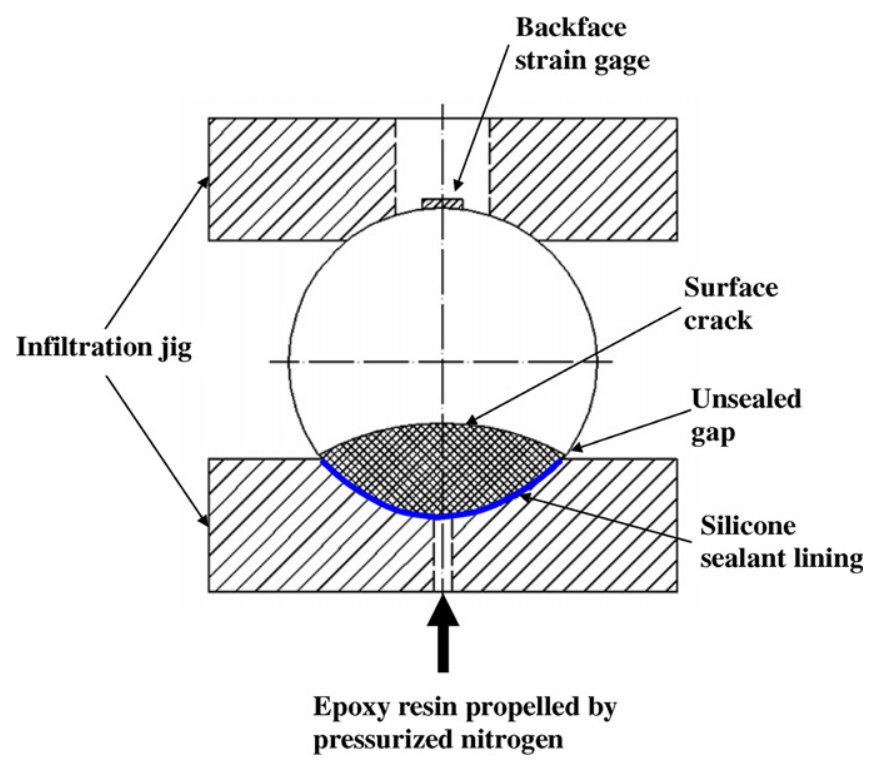

Fig. 1. Nomenclature of the surface crack. from a back face strain gage and a crack tip gage to deduce the interior and the near surface crack closure. The details and validity of this measurement have been discussed elsewhere $[15,16]$. The degree of crack closure is quantified by the ratio $U$ which is the fraction of applied cyclic loading during which the crack stays open. If the stress intensity at which a crack starts to open is $K_{\text {open }}$, the applied stress intensity range is $\Delta K$, then the effective stress intensity range $\Delta K_{\text {eff }}$ for the opened crack and the $U$ value are:

$\Delta K_{\text {eff }}=K_{\max }-K_{\text {open }}$

$U=\Delta K_{\text {eff }} / \Delta K$

where $K_{\max }$ is the stress intensity corresponding to the maximum applied loading $P_{\text {max }}$.

After precracking and growing to about $8 \%$ of the crosssectional area, the test was interrupted. At this stage the crack growth rates at the deepest interior point $\mathrm{A}$ was about $2 \times 10^{-5}$ to $4 \times 10^{-5} \mathrm{~mm} /$ cycle while that at the surface point $G$ was about $4 \times 10^{-5}$ to $6 \times 10^{-5} \mathrm{~mm} /$ cycle in different specimens. The crack was held open at an infiltration load of 0.8 or $0.95 P_{\max }$. Silicone sealant was applied on the rod specimen surface surrounding the crack but care was taken to leave a small portion at the crack tip unsealed. A jig enclosing the surface crack was then installed as shown in Fig. 2. Epoxy resin was propelled through the jig compartment into the crack using pressurized nitrogen at 10 atmospheric pressure. Pressure was gradually reduced when resin was seen dripping out from the unsealed crack tip region. The specimen was held at the infiltration load for a further $18 \mathrm{~h}$ to ensure the resin to become fully hardened. Fatigue testing was then resumed. The mechanical properties of hardened epoxy under compressive loading are listed in Table 1.

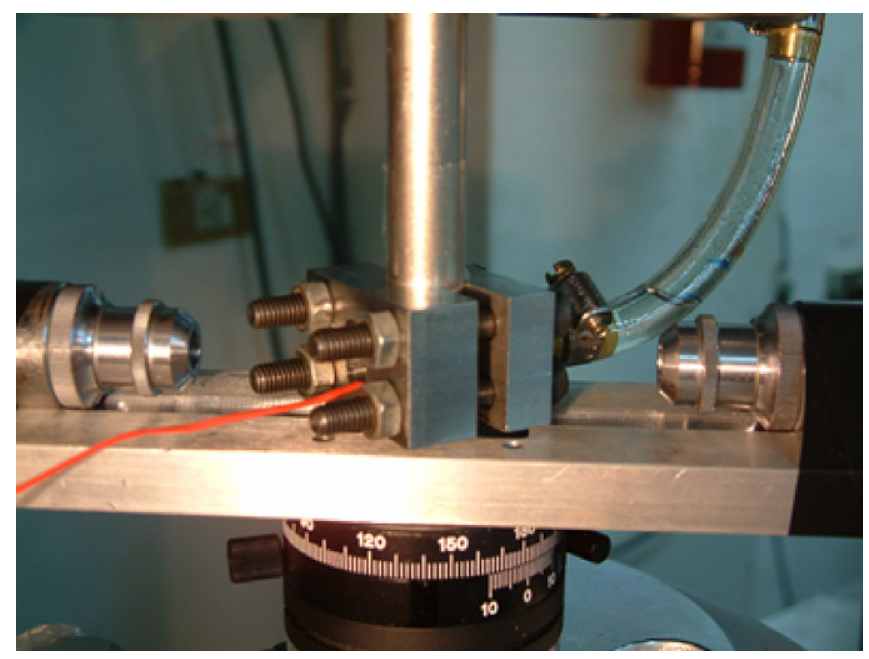

Fig. 2. Schematic and photo of the infiltration set-up.

Table 1

Compressive properties of hardened Epoxy resin

\begin{tabular}{lll}
\hline Compressive strength $(\mathrm{MPa})$ & Modulus $(\mathrm{GPa})$ & Poisson ratio \\
\hline 50.6 & 1.45 & 0.35 \\
\hline
\end{tabular}




\section{Results and discussion}

\subsection{Baseline fatigue crack propagation data}

The fatigue crack propagation rate $\left(\frac{\mathrm{d} a}{\mathrm{~d} N}\right)$ versus the stress intensity range $(\Delta K)$ data derived from five rod specimens [17] can be described by

$\frac{\mathrm{d} a}{\mathrm{~d} N}=1.9 \times 10^{-9}(\Delta K)^{3.502}$

where $\frac{\mathrm{d} a}{\mathrm{~d} N}$ is the crack growth rate in $\mathrm{mm} /$ cycle and $\Delta K$ is in $\mathrm{MPa} \sqrt{\mathrm{m}}$.

When correlated in terms of the effective stress intensity range $\Delta K_{\text {eff, }}$ the baseline crack growth data may be expressed as:

$\frac{\mathrm{d} a}{\mathrm{~d} N}=C_{\mathrm{e}}\left(\Delta K_{\mathrm{eff}}\right)^{m_{\mathrm{e}}}$

With the crack growth rate in $\mathrm{mm} /$ cycle and $\Delta K_{\text {eff }}$ in $\operatorname{MPa} \sqrt{\mathrm{m}}, C_{\mathrm{e}}=8.71 \times 10^{-9}$ and $m_{\mathrm{e}}=3.081$, which were obtained from the best fit to the experimental growth rate data correlated in terms of $\Delta K_{\text {eff }}[17]$.

\subsection{Effect of prop-opening load}

During infiltration, the surface cracks were propped open at $0.8-0.95$ times the maximum load for $18 \mathrm{~h}$. This might affect the subsequent crack growth behavior. To evaluate the extent of this effect, a rod specimen was propped open for $18 \mathrm{~h}$ at 0.95 times the maximum load without giving any infiltration. Fig. 3a shows the crack growth history at the surface point $\mathrm{G}$ and the deepest interior point A of this control specimen. Just before test interruption, the stress intensities were 17.5 and $15.3 \mathrm{MPa} \sqrt{\mathrm{m}}$, respectively. The corresponding growth rates were $\sim 5.1 \times 10^{-5}$ and $3.1 \times 10^{-5} \mathrm{~mm} /$ cycle. On resuming test, the growth rate at the interior dropped slightly and gradually to $2.8 \times 10^{-5} \mathrm{~mm} /$ cycle over a small growth increment before returning to the normal increasing trend. There was no drop in growth rate at the surface over the same interval although a marked slowing down of the increasing trend was evident. As can be seen in Fig. 3a, the rate of increase in crack growth rate is higher at the surface than in the interior, reflecting the fact that the stress intensity gradient is higher at the surface. The slight amount of retardation may probably be overwhelmed by this faster increase in growth rate and so no drop in growth rate was observed there. The corresponding crack closure development is depicted in Fig. 3b. The crack was fully open during the whole loading cycle before the propping load was applied. It remained so for a further amount of growth before starting to develop premature closure at about $15 \%$ of the loading range above the minimum load. These growth and closure phenomena are very similar to that observed in overload retardation [18]. It seems that the extreme stress concentration for prolonged time at the crack tip may bring about similar mechanisms as that in an overload. However, no significant retardation was caused.
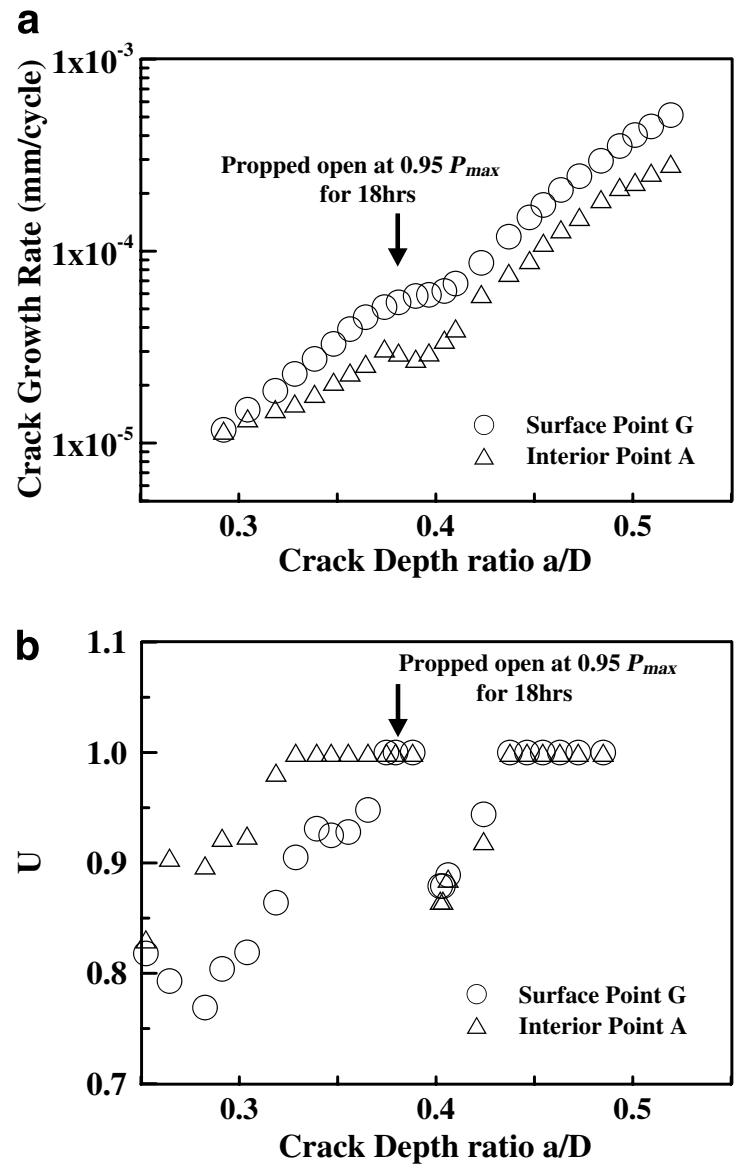

Fig. 3. Effect of prop-opening load on (a) crack growth and (b) crack closure behaviors.

\subsection{Effect of infiltration on crack growth}

Fig. 4a compares the typical crack closure development of an infiltrated specimen at the surface and in the interior. After precracking the $U$ value was in the range $\sim 0.7$ to 0.8 . As the crack continued to grow, $U$ value tended to increase towards unity. Before infiltration, the interior was fully open throughout the whole loading cycle while premature closure still occurred at the surface. Crack closure at the surface was consistently heavier than that in the interior probably due to the fact that the former has less constraint in plastic deformation than the latter. Following infiltration with epoxy, crack closure increased immediately and $U$ dropped to the lowest value of $\sim 0.2$. Unlike that in the pre-infiltration stage, there was no significant difference in closure values between the surface and the interior. This is reasonable as closure is now caused by the infiltrated wedge which is supposed to fill up the crack opening everywhere. Growth rates also showed an immediate drop to the minimum value following infiltration (see Fig. 4b). At the deepest interior point, growth rate decreased from $2.9 \times 10^{-5} \mathrm{~mm} /$ cycle to $1.8 \times 10^{-7} \mathrm{~mm} /$ cycle. At the surface, growth rate dropped from $5.4 \times 10^{-5}$ to $1.43 \times 10^{-6} \mathrm{~mm} /$ cycle. From the maximum retardation point, crack growth gradually accelerated back to the nor- 
a
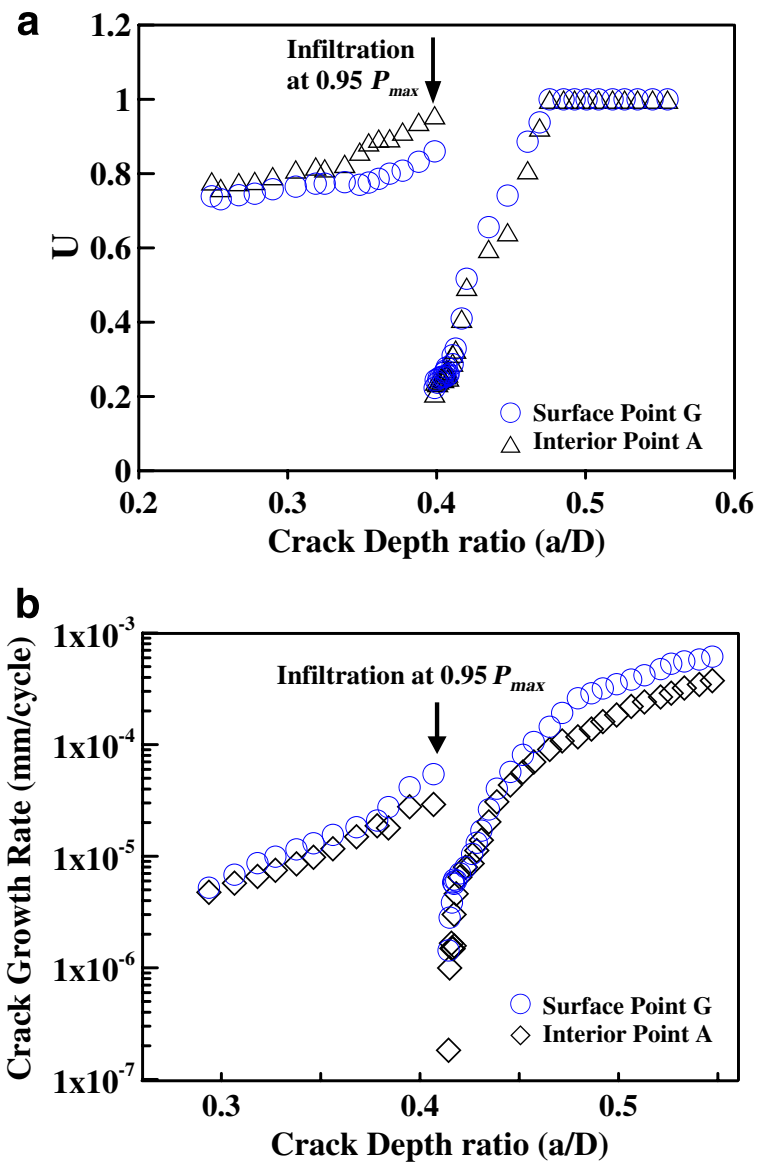

Fig. 4. (a) Crack closure development and (b) crack growth behavior following artificial infiltration at $0.95 P_{\max }$.

mal trend after a crack depth increment of $\sim 1 \mathrm{~mm}$. The infiltration induced retardation was heavier in the interior than at the surface. The reasons are thought to be twofold. Firstly, the amount of drop in closure level was larger in the interior. Secondly, the stress intensity as well as the rate of increase in the stress intensity with crack length were higher at the surface. For the latter reason, the surface growth returned to the normal trend a bit sooner than that in the interior.

\subsection{Effect of infiltration load level}

A test has been carried out with a lower infiltration load level of $0.8 P_{\max }$. Fig. 5 shows that development of its crack closure followed the same trend as that shown under the higher infiltration load. The amount of crack closure induced was smaller and the minimum $U$ value reached following infiltration was $\sim 0.3$. The corresponding amount of induced retardation was also smaller. A comparison of crack growth behavior in the interior and at the surface between the two infiltration levels is shown in Fig. 6a and $\mathrm{b}$, respectively. At the lower infiltration load of $0.8 P_{\max }$, crack growth rate dropped from $3.96 \times 10^{-5}$ to $1.85 \times 10^{-6} \mathrm{~mm} /$ cycle in the interior. At the surface, it dropped from $5.3 \times 10^{-5}$ to $4.57 \times 10^{-6} \mathrm{~mm} /$ cycle. Again

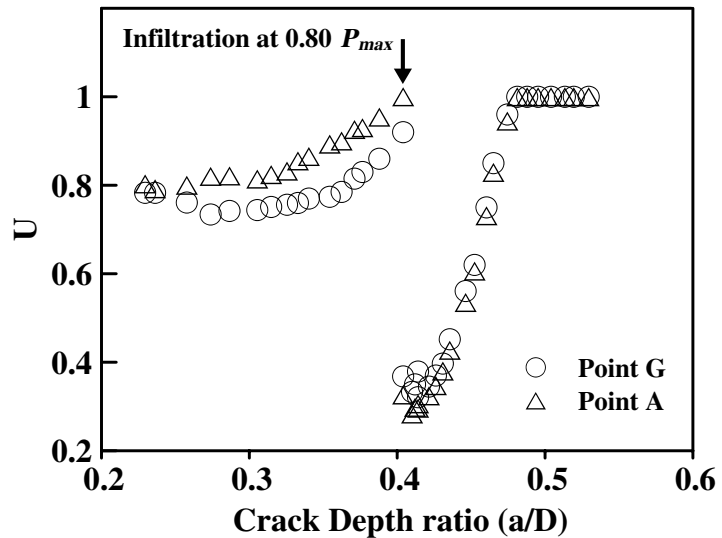

Fig. 5. Crack closure development following artificial infiltration at $0.8 P_{\max }$.

the difference in growth behavior on the surface and in the interior was caused by a combination of differences in the stress intensities and crack closure at these two locations. The dotted lines in Fig. 6 are predictions based on the measured degree of crack closure. They will be
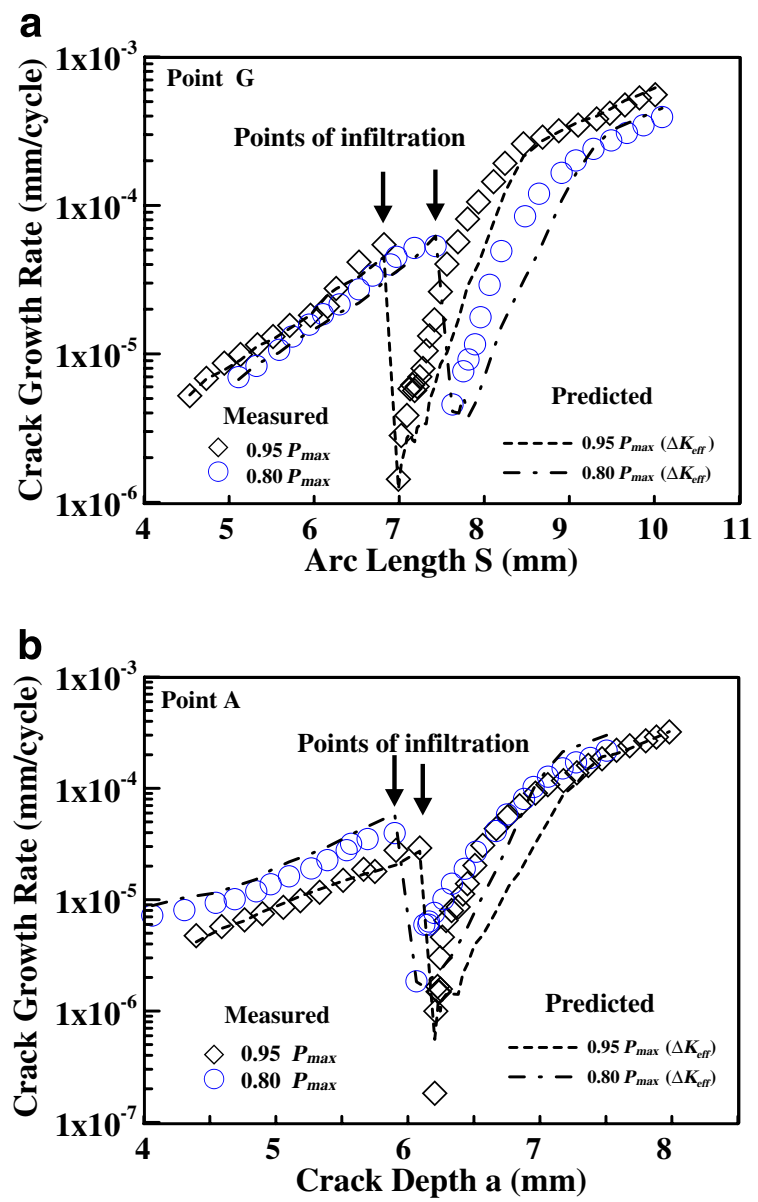

Fig. 6. Crack growth behavior following artificial infiltration under $0.95 P_{\max }$ and $0.8 P_{\max }$. at the (a) surface point $\mathrm{G}$, and (b) deepest interior point A. 
discussed in the later sections and are presented together with the experimental results to avoid redundancy.

\subsection{Reproducibility of the infiltration retardation}

Infiltration tests had been repeated on three different specimens under a prop-opening load of $0.95 P_{\max }$ to evaluate the reproducibility. Owing to the three-dimensional nature of the surface crack and the difficulty in controlling the crack geometry, infiltration could not be carried out under exactly the same conditions in different tests. Instead it was made at roughly the same growth rates $\left(\sim 3 \times 10^{-5} \mathrm{~mm} /\right.$ cycle at the deepest interior point and $\sim 5 \times 10^{-5} \mathrm{~mm} /$ cycle at the surface) while the cracks were of slightly different depths and aspect ratios. Fig. 7a and $\mathrm{b}$ compare the growths at the surface and the interior for these three specimens. At the surface (Fig. 7a), the maximum retardations following infiltration were roughly the same in Tests 1 and 2 while that in Test 3 was smaller. In the interior (Fig. 7b), Test 1 showed the heaviest retardation. This was followed by Test 2 . Test 3 again gave the smallest amount of retardation. Part of the discrepancy among the three tests may be caused by a difference in
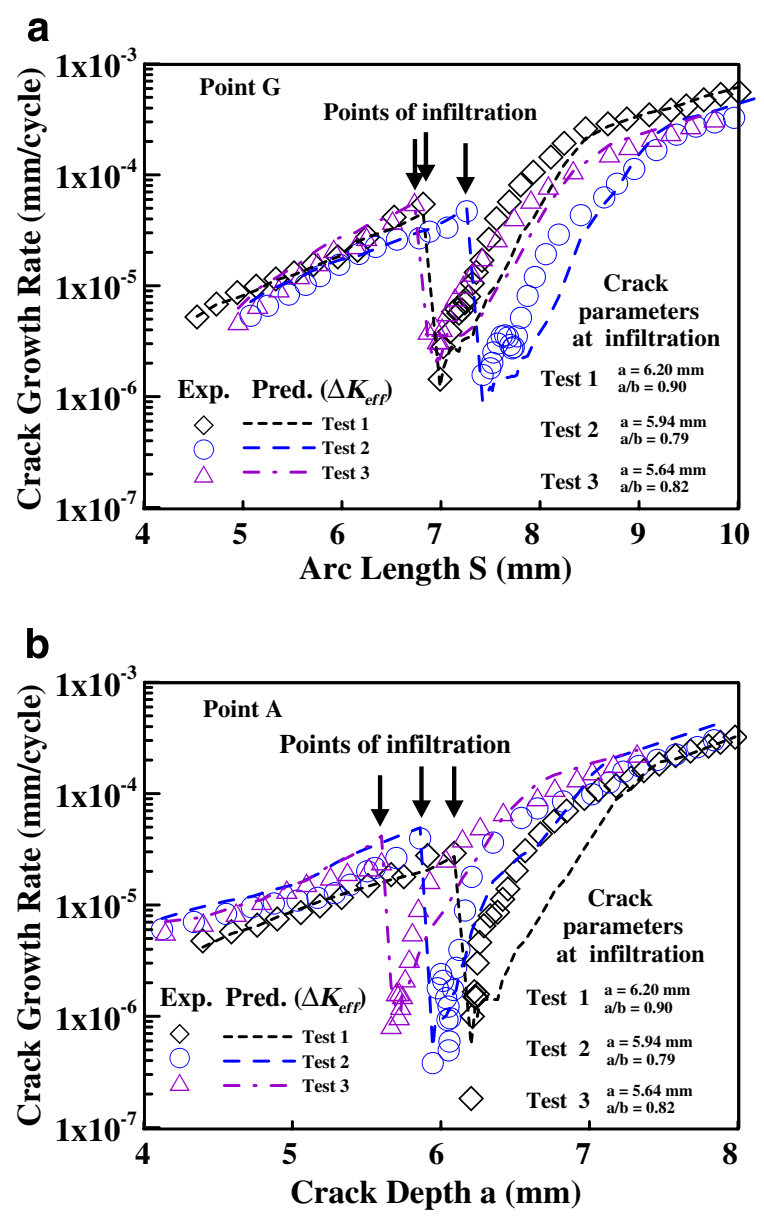

Fig. 7. Crack growth behavior of three surface cracked specimens following artificial infiltration under $0.95 P_{\max }$ at the (a) surface point $\mathrm{G}$, and (b) deepest interior point $\mathrm{A}$. the crack geometries. However, it is believed that the major cause of discrepancy lies in the variation of effectiveness of the infiltration in different specimens.

Fig. 8 shows a pair of matching fracture surfaces of a typical specimen. Two distinct semi-elliptical markings can be seen. The smaller one marks the starter notch. The larger one corresponds to the instantaneous crack front during infiltration. The latter mark was probably formed by the sudden decrease in the crack driving force following infiltration. Epoxy resin can be seen to fill up the crack to the larger semi-elliptical markings. Small bubbles can also be observed scattered in the resin infiltrant. Macroscopically the degrees of penetration of the infiltrant towards the crack front were more or less the same in all three tests. The smaller amount of retardation in Test 3 cannot be accounted for by a lack of infiltrant penetration and may be attributed to the existence of more voids in the resin. In all three tests, although the minimum growth rates
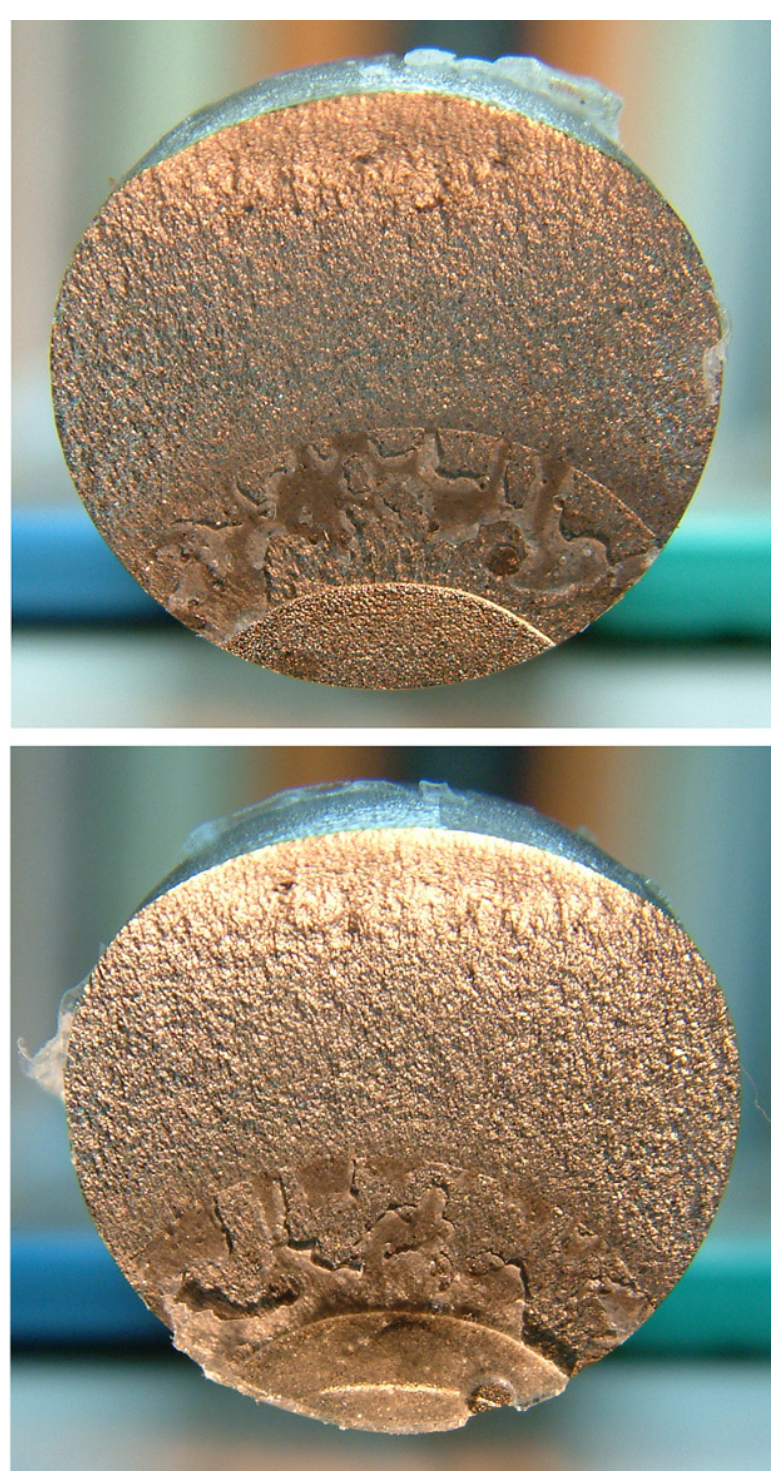

Fig. 8. Fracture surfaces of the broken specimen showing the extent of infiltration. 
were different, the orders of magnitude of the retardation obtained are similar.

\subsection{Prediction of the infiltration induced retardation using crack closure}

With the measured degree of crack closure and the calculated $\Delta K$ at any crack length, the corresponding $\Delta K_{\text {eff }}$ can be deduced. Eq. (4) then allows the crack growth rate to be estimated. The dotted lines in Fig. 6 show such estimations. At both the surface and the interior, the estimated growth rates agreed with the measured rates before infiltration. Just after infiltration, the estimated minimum rates also agreed with that observed at the surface (Fig. 6a). As the growth was recovering from the retardation transient, underestimation in the growth rates occurred. This is commonly seen in other infiltrated retardation [2,5,7-9] and in overload retardation [18] examples. This is thought to be caused by the phenomenon that the extra wedge of material behind the crack tip triggers the closure response but the near tip region is still open. A similar situation was found to occur to a slighter extent in ordinary fatigue cracks and was referred to as "partial crack closure" [19]. On returning to normal growth after the retardation transient, rates estimated from crack closure measurement again agreed with the measured data.

In the interior (Fig. 6b), same behavior as described above is also true for the case where infiltration was made at $0.8 P_{\max }$. For the infiltration load of $0.95 P_{\max }$, the minimum growth rates estimated from crack closure just after infiltration was higher than the observed. Such a phenomenon is contrary to expectation and had never been observed in the other infiltrated retardation examples. Normally, the predicted minimum growth rate would be lower than or equal to the experimentally measured rates $[2,5,7-$ 9]. A lower predicted rates than the measured will occur [2] if the infiltrant does not penetrate sufficiently into the crack tip, giving rise to the "partial crack closure" phenomenon. A possible explanation for the higher predicted rates is the break-down of the crack depth deduction procedure. The current method to deduce the crack depth relied on knowledge of the specimen compliance. Just after infiltration, the unloading compliance was significantly affected by the infiltrated wedge when the applied load fell below the infiltration load. This would cause an underestimation of the crack depth and in turn the growth rate in the interior. The distortion of the specimen compliance lasted for a surface crack length increment of $\sim 0.3 \mathrm{~mm}$. The corresponding crack depth increment is estimated to be within $\sim 0.2 \mathrm{~mm}$. The same complication will not affect the surface crack length measurement and will be less prominent if the infiltrated wedge is of smaller thickness. Incidentally, there is no overestimation in the predicted rates on the surface and also at the lower infiltration load of $0.8 P_{\max }$. In the remaining recovery phase of the retardation transient, marked underestimation again arose presumably due to the partial crack closure effect. The phenomena described above occurred in all other infiltrated specimens as are evident in Fig. 7.

Paris et al. [19] modeled the "partial crack closure" phenomenon by considering a thin parallel faced rigid wedge of thickness $2 h$ being inserted into the crack. The end of this wedge was at a distance $d$ behind the crack tip so that when the crack surfaces came into contact, the extent $d$ was still open. Thus, at the minimum applied load, the crack was subjected to an effective stress intensity of

$K_{\text {min-eff }}=\frac{E h}{\sqrt{2 \pi d}}+\sigma_{\text {nom }} \sqrt{\frac{\pi d}{2}}$

where $E$ was the Young's modulus of the specimen and $\sigma_{\text {nom }}$ was the nominal uniform stress that would be present at minimum load if the crack were absent. Paris et al. [19] argued that when $d$ was small enough that the final contact point of the wedge was within the crack tip stress field, the near tip crack opening displacements was parabolic. In such a case, wedge contact with the crack surface would start to clear from the crack mouth end on loading up. When finally the opening displacement at the tip of the wedge equals the wedge thickness, the crack became "open" according to the conventional sense. This crack opening stress intensity, $K_{\text {open }}$, was related to the crack opening displacement [19]

$K_{\text {open }}=\frac{E h}{2} \sqrt{\frac{\pi}{2 d}}$

By comparing Eqs. (5) and (6), the effective stress intensity at minimum load was found to be roughly equal to $\frac{2}{\pi} K_{\text {open }}$. Based on the above arguments, Paris et al. proposed that the effective crack driving force $\Delta K_{\text {eff corrected }}$ was within the following bounds:

$K_{\max }-\frac{2}{\pi} K_{\text {open }}-\left(1-\frac{2}{\pi}\right) K_{\text {min }} \leqslant \Delta K_{\text {eff corrected }} \leqslant K_{\max }-\frac{2}{\pi} K_{\text {open }}$

Feng et al. [14] found the $\Delta K_{\text {eff corrected }}$ successfully correlated the crack growth retardation caused by artificially wedging open a crack near the notch mouth. In the current case, since the minimum load employed was zero, the upper and lower bounds in Eq. (7) are equal. This corrected effective stress intensity range ( $\left.\Delta K_{\text {eff corrected }}\right)$ may be related to the conventional effective stress intensity range $\left(\Delta K_{\text {eff }}\right.$ defined in Eq. (1)) through

$\Delta K_{\text {eff corrected }}=\left(1-\frac{2}{\pi}\right) K_{\max }+\frac{2}{\pi} \Delta K_{\text {eff }}$

When $\Delta K_{\text {eff corrected }}$ was used to correlate the baseline crack growth data, the $C_{\mathrm{e}}$ and $m_{\mathrm{e}}$ in Eq. (4) became $4.858 \times 10^{-9}$ and 3.248, respectively. The results of using this corrected effective stress intensity to predict the retardation responses in two typical infiltration tests are shown in Fig. 9. It can be seen that by using $\Delta K_{\text {eff corrected, growth rate underestima- }}$ tion in the recovery phase has been greatly improved and in fact the predicted and measured rates overlapped in three of the four cases. However, the amounts of maximum 
retardation are smaller than the observed by nearly an order of magnitude. A higher predicted rates than observed persisted for about $0.5 \mathrm{~mm}$. It should be noted that the correction factor $2 / \pi$ on $K_{\text {open }}$ was derived assuming a parallel faced wedge made of rigid material. The current situation just after the infiltration differs from Paris et al.'s partial crack closure model in at least two aspects: (i) the infiltrated wedge is far from being rigid; (ii) the shape of the wedge takes up the crack opening profile and has variable thicknesses along the crack depth. The first aspect makes the stress intensity of the crack at minimum load differs from that dictated by Eq. (5). The exact value of this intensity may be computed by considering a distribution of variable crack face contact pressure which magnitude depends on the deformation and reaction of the wedge at each location. The second aspect suggests that different points on the infiltrated wedge get clear from the crack surface at more or less the same instance. This implies that the $K_{\text {open }}$ measured should correspond to the stress intensity caused by the variable crack face contact pressure. In other words, instead of the $\Delta K_{\text {eff corrected }}$ of Eq. (7), the measured $K_{\text {open }}$ was more representative of the minimum effective stress intensity in an infiltrated crack. Thus the growth rates pre-
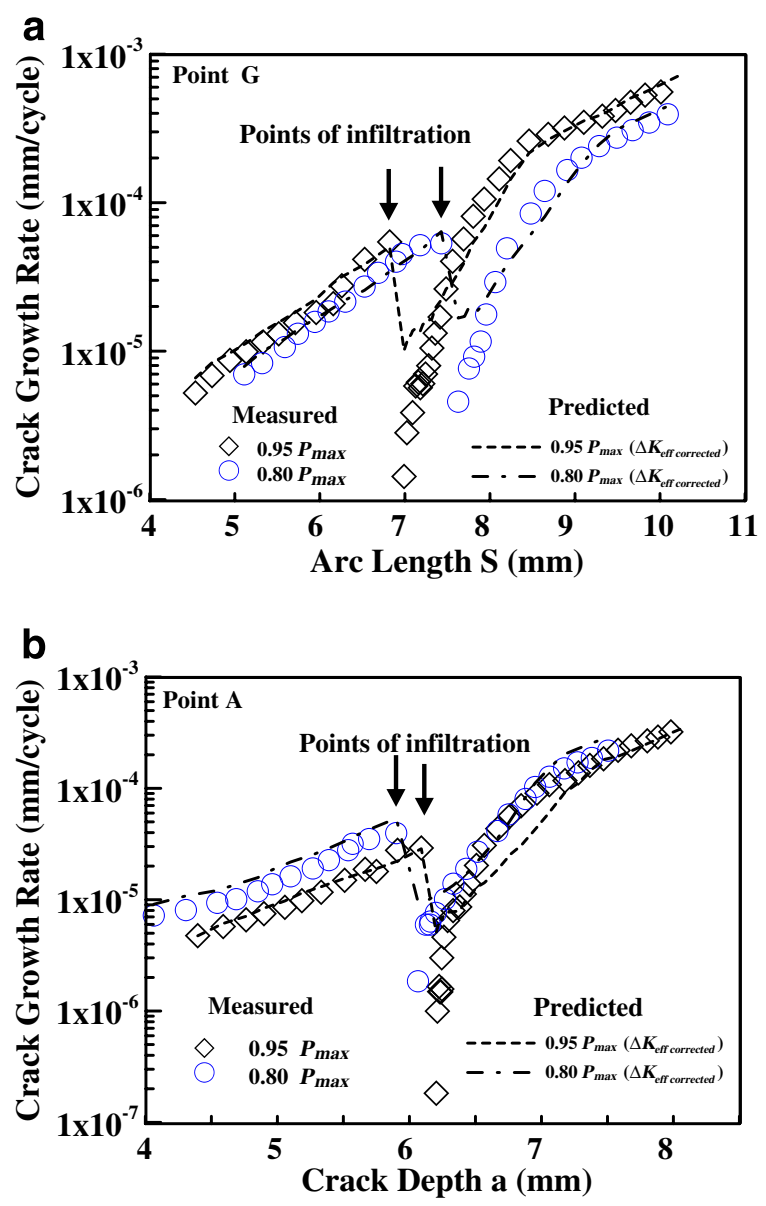

Fig. 9. Crack growth behavior following artificial infiltration under $0.95 P_{\max }$ and $0.8 P_{\max }$ predicted using $\Delta K_{\text {eff corrected }}$ at the (a) surface point $\mathrm{G}$ and $(\mathrm{b})$ deepest interior point $\mathrm{A}$. dicted from the measured closure are close to the observed growth rate just after infiltration. As the crack grew longer, the above two aspects gradually gave way and the situation might be getting closer to the partial crack closure model described by Paris et al. Hence the predictions from $\Delta K_{\text {eff corrected }}$ fall in lines with the observed data $0.5 \mathrm{~mm}$ away from the infiltration point. It is reasoned that a model such as that employed in Ref. [13] which computed the crack opening stress intensities from the variable wedge contact pressure may give a better overall prediction of the retardation behavior following infiltration. Work in that direction is underway.

\section{Conclusion}

A method for infiltrating polymeric resin into a surface crack has been established. It has been demonstrated by filling up the crack flanks with resin at $80 \%$ of the maximum cyclic loading, a maximum crack growth retardation of more than one order of magnitude can be achieved at the surface and in the interior. On filling at $95 \%$ of the maximum cyclic loading, maximum retardation of around two orders of magnitude can be obtained. The extent of crack growth affected by the retardation transient is $\sim 1 \mathrm{~mm}$ in the interior and $\sim 1.5 \mathrm{~mm}$ on the surface. The measured degree of crack closure can give a good estimation of the retardation behavior in the vicinity of the infiltration point but it tends to predict a lower than observed growth rates during the remaining recovery phase of the retardation. On the other hand, a corrected effective stress intensity range based on Paris et al.'s partial crack closure model gives a good prediction of growth behavior in that remaining portion of the recovery phase while it significantly underestimated the amount of maximum retardation.

\section{Acknowledgement}

The authors are grateful to the National Science Council, ROC for financial support for this work through the projects NSC93-2212-E-002-062 and NSC95-2212-E-002014.

\section{References}

[1] Shin CS, Hsu SH. Fatigue life extension by an artificially induced retardation mechanism. Eng Fract Mech 1992;43(4):677-84.

[2] Shin CS, Wang CM, Song PS. Fatigue damage repair - a comparison of some possible methods. Int J Fatigue 1996;18(8):535-46.

[3] UR-Rehman A, Thomason PF. The effect of artificial fatigue crack closure on fatigue crack growth. Fat Fract Eng Mater Struct 1993;16(10):1081-90.

[4] Sharp PK, Clayton JQ, Clark G. Retardation and repair of fatigue cracks by adhesive infiltration. Fat Fract Eng Mater Struct 1997;20(4):605-14

[5] Shin CS, Huang KC, Li RZ. Artificial retardation of fatigue crack growth by the infiltration of cracks by foreign materials. Fat Fract Eng Mater Struct 1998;21:835-46.

[6] Song PS, Wang CM, Shin CS. An investigation on various infiltration methods for fatigue crack repair. In: Proceedings of the 1994 Far East 
Conference on NDT (FENDT '94) and ROCSNT Ninth Annual Conference, Taipei, ROC, 1994:221-8.

[7] Sheu BC, Song PS, Shin CS. The effect of infiltration induced crack closure on crack growth retardation. Scripta Metallurgica et Materialia 1994;31(10):1301-6.

[8] Song PS, Hwang S, Shin CS. Effect of artificial closure materials on crack growth retardation. Eng Fract Mech 1998;60(1):47-58.

[9] Shin CS, Chen ZZ. Fatigue life extension by electroless nickel infiltration plating. Int J Fatigue 2001;23:777-88.

[10] Brown EN, White SR, Sottos NR. Retardation and repair of fatigue cracks in a microcapsule toughened epoxy composite-Part I: Manual infiltration. Compos Sci Technol 2005;65:2466-73.

[11] Wang YY, Zho MX, Feng DQ. A study of retarding fatigue crack growth using an artificial wedge. Fat Fract Eng Mater Struct 1993;16(3):363-76.

[12] UR-Rehman A. An investigation of methods of reducing fatigue crack growth by artificial crack-closure effects. Ph.D. thesis, Department of Aeronautical and Mechanical Engineering, University of Salford:1992.
[13] Shin CS, Cai CQ. A model for evaluating the effect of fatigue crack repair by the infiltration method. Fat Fract Eng Mater Struct 2000;23:835-45.

[14] Feng M, Ding F, Jiang Y. A study of crack growth retardation due to artificially induced crack surface contact. Int J Fatigue 2005;27: 1319-27.

[15] Shin CS, Cai CQ. A normalized area-compliance method for monitoring the surface crack development in a cylindrical rod. Int $\mathbf{J}$ Fatigue 2005(27-7):801-9.

[16] Shin CS, Cai CQ. Experimental and finite element analyses on stress intensity factors of elliptical surface crack in a circular shaft under tension and bending. Int J Fract 2004(129):239-64.

[17] Shin CS, Cai CQ. Evaluating fatigue crack propagation properties using a cylindrical rod specimen. Int J Fatigue 2007;29:397-405.

[18] Shin CS, Hsu SH. On the mechanism and behaviour of overload retardation in AISI 304 stainless steel. Int J Fatigue 1993;15(3): 181-92.

[19] Paris PC, Tada H, Donald JK. Service load fatigue damage-a historical perspective. Int J Fatigue 1999;21:S35-46. 